\title{
Contrast Enhancement of Long-Range Periodic Structures using Hole-Free Phase Plate.
}

\author{
Hirofumi Iijima ${ }^{1}$, Yuji Konyuba ${ }^{1}$, Naoki Hosogi ${ }^{1}$, Yoshihiro Ohkura ${ }^{1}$, Hiroshi Jinnai ${ }^{2}$ and Takeshi \\ Higuchi $^{2}$ \\ ${ }^{1 .}$ EM Business Unit, JEOL Ltd., 3-1-2 Musashino, Akishima, Tokyo 196-8558, Japan \\ 2. Institute of Multidisciplinary Research for Advanced Materials (IMRAM), Tohoku University, 2-1-1 \\ Katahira, Aoba-ku, Sendai 980-8577, Japan.
}

Phase contrast transmission electron microscopy is a powerful tool to enhance the image contrast of transparent materials such as ice-embedded biological specimens and polymer materials. In this method, a phase plate, which is placed at the back-focal plane of the objective lens, gives a phase shift for scattered electron waves, resulting in a significant enhancement contrast of specimens in images. Zernike phase plate (ZPP), consisting of a thin carbon film with a small central hole, is first tested practical phase plate [1]. However, ZPP has disadvantages for image quality. Phase contrast of specimens in low spatial frequency does not improve, since the scattered electron passing through a central hole of ZPP does not change their phases. The threshold frequency at the center hole edge is called cut-on frequency. The additional disadvantageous effect of the abrupt cut-on frequency reveals that strong fringes appear.

Hole-free phase plate (HFPP) is one of other types of phase plate, consisting only of thin carbon film. In HFPP, the charging on a carbon film due to high-density electron beam (cross-over) acts like a small central hole of ZPP. The size of cross-over on HFPP is smaller than typical size of a central hole of ZPP, which is typically $1 \mu \mathrm{m}$ in diameter. Thus, the cut-on frequency of HFPP is lower than ZPP and phase contrast of larger structures is enhanced by HFPP. Also with HFPP, The fringing effects, which is observed in ZPP, reduces because the width of the fringes is increasing to be wider than sample size with the very small cut-on frequency.

In this study, we applied the HFPP to enhance the phase contrast for unstained polymer materials. A $200 \mathrm{kV}$ field-emission electron microscope (JEM-2200FS) equipped with Schottky electron source was used to obtain a small cross-over on a HFPP. A HFPP, which is made of $15 \mathrm{~nm}$-thick amorphous carbon, is set on the back focal plane of the objective lens of focal length $2.8 \mathrm{~mm}$.

Figure 1 compares conventional TEM and HFPP-TEM images for $50 \mathrm{~nm}$-thick unstained polymer section (Polystyrene-b-isoprene, Polymer Source Inc.). The conventional TEM images have low contrast, even with the large defocus (Figure 1 (a), (b)). In the HFPP-TEM image, lamellar structures of the polymer are clearly observed (Figure 1 (c)).

Figure 2 shows the diffractogram of HFPP-TEM image in Figure 1. Peaks in the diffractogram are corresponding to $0.02 \mathrm{~nm}^{-1}$ in reciprocal space, indicating the lamellar period of specimen is $50 \mathrm{~nm}$ (Figure 2). A reason for that such a long periodic structure can be observed by the HFPP-TEM is that the cross-over on a HFPP, which is supposed to be a phase modulation region, is small enough to enhance the low-spatial frequency of the lamellar structures.

Figure 3 shows an intensity profile of a cross-over spot, which is formed on a back focal plane and observed in diffraction mode. The measured size of a cross-over on a HFPP in real space (scale in lower 
bar) is approximately $120 \mathrm{~nm}$ in full width at half maximum (FWHM) calibrated with the focal length for the objective lens. The radius in reciprocal space (scale in upper bar) is less than $0.02 \mathrm{~nm}^{-1}$, which is supposed to be cut-on frequency and is lower than the frequency of lamella structure.

In conclusion, we have successfully observed the polymer lamella structure with good contrast by phase contrast microscopy using a HFPP. We also measure the size of cross-over on the HFPP experimentally. From the size, we can judge that the cut-on frequency for HFPP is lower than $0.02 \mathrm{~nm}^{-1}$, which is corresponding to $50 \mathrm{~nm}$.

References:

[1] R. Danev et al, J. Phys. Sci. Jpn. 70 (2001) p. 696.

[2] M. Malac et al, Ultramicroscopy 118 (2012) p. 77.
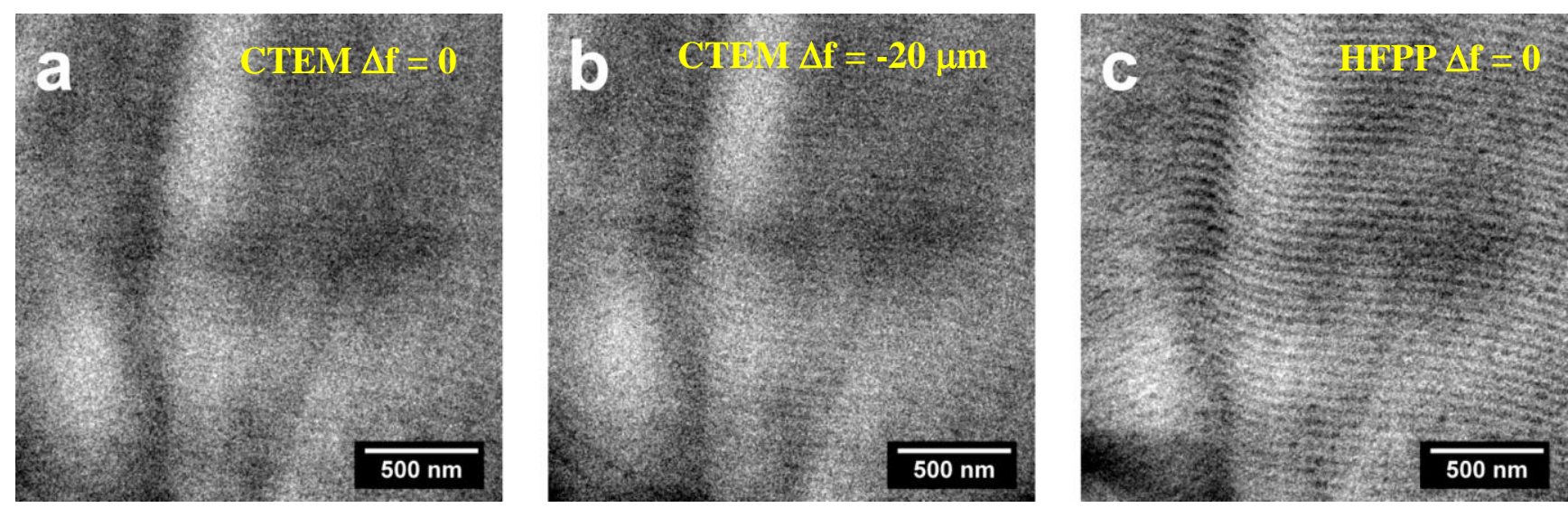

Figure 1. Conventional TEM and HFPP-TEM images of unstained polymer. (a) Conventional TEM taken at just-focus, (b) conventional TEM taken at $-20 \mu \mathrm{m}$ defocus, and (c) HFPP-TEM taken at justfocus.

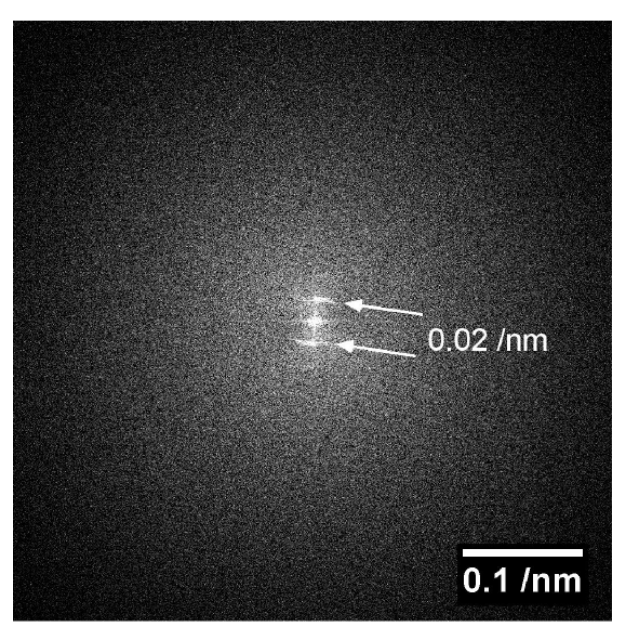

Figure 2. Diffractogram of HFPPTEM image in Figure 1 (c).

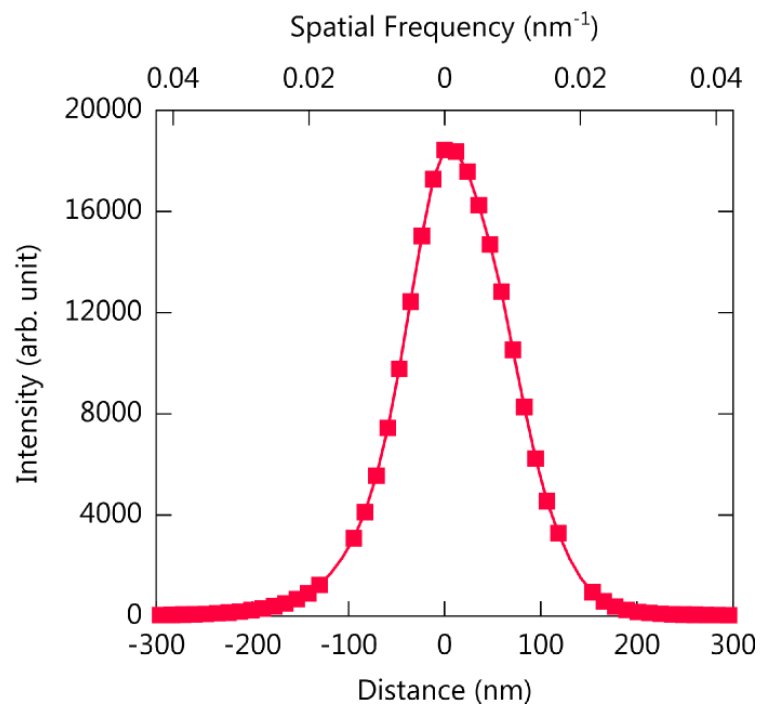

Figure 3. Intensity profile of a crossover on a HFPP. 